\title{
QUANDO FINİ IL COLONIALISMO ITALIANO? TRE DECOLONIZZAZIONI A CONFRONTO
}

\author{
ANTONIO M. MORONE (*) \\ Nota presentata dal m.e. Silvio Beretta \\ (Adunanza del 28 marzo 2019)
}

SUNTO. - La fine del colonialismo italiano non può semplicemente essere confinata alla sconfitta militare dell'Italia durante la seconda guerra mondiale e alla conseguente perdita del possesso sulle colonie. Infatti l'Italia repubblicana rivendicò con forza la restituzione delle colonie fino alla bocciatura alle Nazioni Unite del Compromesso BevinSforza nel 1949. È dunque legittimo parlare di un colonialismo italiano di epoca repubblicana, con suoi caratteri specifici, che fu la premessa di un processo di decolonizzazione diverso per Libia, Eritrea e Somalia. La presente nota prende in considerazione la specificità dei tre percorsi di decolonizzazione in riferimento al passaggio da una rinnovata politica coloniale italiana a una più propriamente postcoloniale.

$* * *$

ABSTRACT. - The end of Italian colonialism cannot simply be limited to Italy's military defeat during the Second World War and the consequent loss of colonial possessions. In actuality, the Republic of Italy clamored for the restitution of the former colonies until the rejection of the Bevin-Sforza Compromise at the U.N. General Assembly in 1949. It is therefore justifiable to point out the reality of an Italian colonialism during the Republican era, with its specific characteristics, which was the premise of the decolonization processes for Libya, Eritrea and Somalia. This note takes into consideration the specificity of these three paths of decolonization with reference to the Italian transition from a renewed colonial policy to a real postcolonial one.

L'Italia perse il possesso delle colonie africane durante la seconda guerra mondiale a causa della sconfitta subita in battaglia contro le trup-

(*) Dipartimento di Scienze Politiche e Sociali, Università degli Studi di Pavia, Italy. E-mail: antmorone@hotmail.com 
pe inglesi e del Commonwealth. A cadere per prima fu l'Africa orientale italiana (Aoi): Mogadiscio venne occupata il 27 febbraio, Asmara il $1^{\circ}$ aprile e Addis Abeba il 5 aprile, mentre l'ultima resistenza italiana del duca Amedeo D'Aosta sull'Amba Alagi finì il 17 maggio. Sul fronte Nord africano, la Cirenaica venne definitivamente occupata nel novembre 1942 dalle truppe inglesi che il 23 gennaio 1943 entrarono a Tripoli, mentre il Fezzan cadde sotto il controllo militare della "Francia libera". La conclusione della guerra invece di portare a una rapida sistemazione delle ex colonie italiane, diede il via a una lunga e complessa vicenda politica e diplomatica che si protrasse fino alla fine degli anni Quaranta. L'articolo 23 del Trattato di pace firmato a Parigi il 10 febbraio 1947 imponeva all'Italia la rinuncia alle colonie, ma non stabiliva affatto la loro definitiva sistemazione, rinviando ogni decisione alle Quattro potenze vincitrici (Stati Uniti d'America, Unione Sovietica, Francia e Gran Bretagna) che avevano un anno di tempo dalla ratifica del Trattato medesimo per raggiungere una soluzione, viceversa il dossier coloniale sarebbe passato all'Organizzazione delle Nazioni Unite (Onu), come poi in effetti accadde nel settembre 1948. La firma del Trattato per parte italiana non corrispose affatto a una sua reale messa in pratica per quel che riguarda le ex colonie. L'Italia aveva infatti perso il suo status di potenza coloniale, vivendo questa diminutio del suo ruolo internazionale come una vessazione, un'ingiustizia, alla quale la nuova classe dirigente repubblicana cercò di porre rimedio, rivendicando il ritorno in colonia, per tutto il lungo dopoguerra fino al 1949. A contrastare con l'idea di una possibile «riconsegna delle colonie all'Italia» giocarono molti fattori: L'ostilità degli arabi, l'opposizione strenua dell'Etiopia e, soprattutto, la scarsa affidabilità dell'Italia finché non fosse stato precisato il suo orientamento politico e di conseguenza il suo posto nel sistema internazionale». ${ }^{1}$ La vicenda della sistemazione delle ex colonie italiane si avviò a una rapida soluzione solo sul finire degli anni Quaranta, quando venne raggiunto un accordo in due tempi all'Assemblea generale dell'Onu dopo che il cosiddetto Compromesso Bevin-Sforza risultò impraticabile: la risoluzione n. 289 del 21 novembre 1949 dispose che la Somalia fosse affidata all'Italia in amministrazione fiduciaria per 10 anni, mentre per la Libia prevalse una soluzione favorevole alla sua piena indipendenza, proclamata il 24 dicem-

1 G.P. Calchi Novati (a cura di), L'Africa d'Italia. Una storia coloniale e postcoloniale, Roma, Carocci, 2011, pp. 360-1. 
bre 1951; la risoluzione n. 390 del 2 dicembre 1950 dispose invece che l'Eritrea divenisse "unità autonoma" federata all'Impero etiopico. Dopo una fase preparatoria sotto la supervisione delle Nazioni Unite, la federazione tra Etiopia ed Eritrea entrò in vigore il 15 settembre 1952, mentre l'Italia ritornò in Somalia il $1^{\circ}$ aprile 1950 con il preciso mandato di preparare l'ex colonia all'indipendenza nell'arco di un decennio.

La storiografia sul colonialismo italiano, per tutta quella stagione che andò dagli anni Settanta del Novecento fino alla fine del secolo, fu fortemente impegnata nel rileggere in chiave critica la prima e precedente stagione di indagini e ricerche che iniziò in diretta continuità con gli studi coloniali e fu fortemente condizionata da una visione apologetica e sostanzialmente positiva del ruolo giocato dal colonialismo nella storia delle società colonizzate. ${ }^{2} \mathrm{Nel} 1964$, il colonialismo era definito come «la forma più blanda di dominazione che mai nella storia un popolo abbia esercitato su un altro» nelle parole di Carlo Giglio, che aveva insegnato Storia e politica coloniale prima della guerra ed era poi rimasto in cattedra insegnando Storia e istituzioni dei paesi afro-asiatici all'Università degli Studi di Pavia fino alla morte improvvisa nel 1976. ${ }^{3}$ Quella di Giglio non fu una voce isolata, ma piuttosto una tra quelle più rappresentative di un'intera scuola all'interno dell'Accademia italiana che si fece portatrice di un'interpretazione positiva e acritica del passato coloniale dell'Italia. La revisione critica della storia coloniale intrapresa durante la seconda stagione di studi fu lunga e laboriosa e passò per la (ri)definizione dell'oggetto stesso di studio. I processi e le istituzioni coloniali, oltre alla politica e alle relazioni internazionali nel cui ambito si svolse la vicenda coloniale furono indagati soprattutto con riferimento alla fase iniziale della conquista dei possedimenti d'oltrema-

2 Per una ricostruzione critica della storiografia sul colonialismo italiano si veda M. Lenci, Dalla storia coloniale alla storia dell'Africa, Africa: rivista trimestrale di studi e documentazione dell'Istituto italo-africano, 58, 3 (2003), pp. 207-218; G.P. Calchi Novati (a cura di), Il colonialismo e l'Africa. L'opera storiografica di Carlo Giglio, Roma, Carocci, 2004; N. Labanca, Il passato coloniale come storia contemporanea, in A. Del Boca, I gas di Mussolini. Il fascismo e la guerra d'Etiopia, Roma, Editori Riuniti, 2007, pp. 7-26; A.M. Morone, L'Italie et l'héritage du colonialisme. Entre recherches et débat public, in A. Hammouche, G. Meynier, R. Pfefferkorn (sous la direction de), Colonial, Postcolonial, Décolonial, Raison Présente, 199, 3 (2016), pp. 23-33.

3 C. Giglio, Colonizzazione e decolonizzazione, Cremona, Mangiarotti,1964, p. 141. 
re per poi analizzare la costruzione del mondo coloniale fino alla seconda guerra mondiale. Alla fase finale del colonialismo e alla transizione verso l'indipendenza fu invece dedicata una attenzione più limitata e circoscritta principalmente ai lavori di Angelo Del Boca, Gian Paolo Calchi Novati, Nicola Labanca e Gianluigi Rossi. ${ }^{4}$

A partire dagli anni Duemila una terza stagione storiografica ha insistito, molto più di quanto si fosse fatto prima, sul superamento della storia del colonialismo italiano intesa come storia dell'espansione coloniale, ossia degli italiani in Africa, aprendo alla storia dell'Africa e a una interpretazione della vicenda coloniale intesa come storia dell'Italia e dell'Africa, degli italiani e degli africani. L'approccio proposto dall'africanistica non è stato affatto generalmente condiviso, né adottato unanimemente negli ultimi anni, ma ha senza dubbio generato confronti e discussioni proficue nel rapporto con il più tradizionale approccio dei contemporaneisti o degli internazionalisti, attenti soprattutto alla dimensione italiana e diplomatica del colonialismo. Va poi dato merito di aver contribuito a svecchiare gli studi sul colonialismo italiano anche a un altro approccio decisamente innovativo come quello dei Postcolonial Studies approdati recentemente al caso italiano. In questa terza stagione di studi si è andato accentuando anche l'interesse per la fine del colonialismo, le continuità ed eredità coloniali e le memorie del passato dominio italiano in Africa. Inoltre, gli studi sul colonialismo italiano hanno registrato una rapida internazionalizzazione con l'uscita a metà degli anni Duemila dei primi volumi collettanei in lingua inglese dove dialogavano contributi di ricercatori italiani, africani e internazionali, mentre i filoni di ricerca tradizionalmente più indagati come quelli della storia politica, diplomatica o militare furono progressivamente arricchiti da lavori di storia culturale e sociale. ${ }^{5}$ I risultati complessivi furono sicuramente lusinghieri se Roberta Pergher nel 2007 sottolineava come di fronte al crescente interesse

4 In particolare A. Del Boca, Gli Italiani in Africa orientale, vol. 4, Nostalgia delle colonie, Roma-Bari, Laterza, 1984; Id., Gli italiani in Libia, vol. 2, Dal fascismo a Gheddafi, Roma-Bari, Laterza, 1986; G.P. Calchi Novati, Il Corno d'Africa nella storia e della politica. Etiopia, Somalia e Eritrea fra nazionalismi, sottosviluppo e guerra, Torino, Sei, 1994; N. Labanca, Oltremare. Storia dell'espansione coloniale italiana, Bologna, il Mulino, 2002; G. Rossi, La questione delle ex colonie italiane dopo il trattato di pace: 1947-1949, Milano, Giuffrè, 1980.

5 Si veda P. Palumbo (ed.), A Place in the Sun. Africa in Italian Colonial 
scientifico per la storia del colonialismo italiano «non è più giustificata» quell'idea di una «carenza di ricerche e discussioni» che per lungo tempo aveva caratterizzato la storiografia precedente. ${ }^{6}$ Rimane al contrario vera la scarsa capacità degli studi sul colonialismo italiano di incidere sulla più generale riflessione storiografica sull'Italia contemporanea. Con riferimento specifico alla fine del colonialismo, che qui è in discussione, fa riflettere la mancanza di una riflessione sul colonialismo in uno dei più recenti e aggionnati volumi pubblicati da Guido Formigoni e Daniela Saresella sulla transizione italiana nel dopoguerra: si dà giustamente conto della transizione al mondo bipolare, della transizione alla democrazia e di quella sociale verso il cosiddetto "miracolo economico", limitandosi a un paio di accenni al mito del "buon italiano" e ai suoi riflessi coloniali. ${ }^{7}$ Finisce per essere un paradosso come la vicenda del colonialismo italiano sia da tempo studiata dalle storiografie dei paesi che furono un tempo colonie italiane o da più ampie reti di ricerca internazionale e ormai da diverse generazioni di studiosi italiani, ma continui a essere marginale nella più generale ricostruzione e analisi della storia d'Italia.

La ricchezza di ricerche e di approcci disciplinari che negli ultimi anni ha sempre più caratterizzato lo studio del colonialismo italiano e delle sue continuità postcoloniali è bene sintetizzata dall'obiettivo che si prefiggono i due curatori del volume di recente pubblicazione Quel

Culture from Post-Unification to the Present, Berkeley, University of California Press, 2003. Per le due curatrici di un altro celebre volume, l'obiettivo era quello di contribuire a «una maggiore internazionalizzazione» degli studi: R. Ben-Ghiat, M. Fuller (eds.), Italian Colonialism, New York, Palgrave Macmillan, 2005, p. 1. Occorreva invece studiare «come l'eredità del colonialismo continua ad avere un impatto sul presente» per contribuire a un «dibattito postcoloniale che in Italia è piuttosto arretrato» per J. Andall, D. Duncan (eds.) Italian colonialism. Legacy and Memory, Bern, Peter Lang, 2005, p. 10.

6 R. Pergher, Impero immaginato, impero vissuto. Recenti sviluppi nella storiografia del colonialismo italiano, «Ricerche di Storia Politica», 1 (2007), p. 53.

7 G. Formigoni, D. Saresella (a cura di), 1945. La transizione del dopoguerra, Roma, Viella, 2017, pp. 207, 279, 284 dove si rimanda al noto volume di A. Del Boca, Italiani brava gente?, Vicenza, Neri Pozza, 2005. Più attento invece alla dimensione coloniale della transizione italiana del secondo dopoguerra è invece il fascicolo monografico de Il Politico, Italia in transizione: il secondo dopoguerra, a cura di S. Beretta, 246, settembre-dicembre 2017, che ospita il contribuito di A. Pes, Il lavoro italiano in colonia nel dibattito politico tra il 1946 e il 1949, pp.160-175. 
che resta dell'impero: occorre «scardinare i limiti temporali del colonialismo italiano - scrivono Valeria Deplano e Alessandro Pes - e analizzare il suo impatto sull'Italia dall'età liberale a quella contemporanea, così da riconsiderare come la cultura coloniale abbia influito e continui ad influire sulla cultura nazionale». ${ }^{8}$ Proprio l'enfasi sull'eredità del colonialismo impone, a giudizio di chi scrive, la necessità di ritornare a interrogarsi sulla fine del colonialismo con l'obiettivo di distinguere appunto la fase terminale della storia del dominio coloniale italiano, dalle sue memorie e dalle sue conseguenze o eredità di più o meno lungo periodo per l'Italia e i paesi africani che un tempo furono colonie. Parlando di continuità coloniali, il rischio può essere quello di dilatare all'infinito il concetto di colonialismo, finendo per svuotarlo di un chiaro significato storico: il colonialismo «distinto dalle istituzioni attraverso cui il potere coloniale è esercitato finisce appunto per far apparire il colonialismo ovunque e quindi da nessuna parte»." Proprio i Postcolonial Studies applicati al caso italiano, ${ }^{10}$ che hanno senza dubbio avuto negli ultimi anni il grande merito di «correggere forme di amnesia o progetti sospetti di revisionismo storico» in relazione al passato coloniale, ${ }^{11}$ scontano tuttavia il limite di essersi concentrati sulle continuità nel contesto dell'ex madrepatria con il rischio di «obliterare la storia dei

8 V. Deplano, A. Pes (a cura di), Quel che resta dell'impero. La cultura coloniale degli italiani, Milano-Udine, Mimesis,2014, pp. 12-13.

9 F. Cooper, Colonialism in Question: Theory, Knowledge, History, Berkeley, University of California Press, 2005, p. 47.

10 M. Colin, E. R. Laforgia (sous la direction de), L'Afrique coloniale et postcoloniale dans la culture, la littérature et la société italiennes. Représentations et témoignages, Caen, Presses universitaire de Caen, 2003; S. Mezzadra, La condizione postcoloniale : storia e politica nel presente globale, Verona, Ombre corte, 2008 ; G. Proglio, Memorie oltreconfine: la letteratura postcoloniale italiana in prospettiva storica, Verona, Ombre Corte, 2011; C. Lombardi-Diop, C. Romeo (eds.), Postcolonial Italy. Challenging National Homogeneity, New York, Palgrave Macmillan, 2012; G. Giuliani, C. Lombardi-Diop, Bianco e Nero. Storia dell'identità razziale degli italiani, Firenze, Le Monnier, 2013; F. Sinopoli (a cura di), Postcoloniale italiano. Tra letteratura e storia, Roma, Novalogos, 2013; G. Giuliani (a cura di), Il colore della nazione, Firenze, Le Monnier, 2015; G. Grechi, V. Gravano (a cura di), Presente imperfetto. Eredità coloniali e immaginari razziali contemporanei, Milano-Udine, Mimesis, 2016; G. Giuliani, Race, Nation and Gender in Modern Italy. Intersectional Representations in Visual Culture, New York, Palgrave Macmillan, 2018.

11 S. Ponzanesi, The Postcolonial Turn in Italian Studies. European Perspectives, in Lombardi-Diop, Romeo (eds.), Postcolonial Italy, cit., p. 59. 
popoli che vissero in quelle che divennero colonie», come ha scritto Frederick Cooper per altri contesti postcoloniali. ${ }^{12}$ Non si tratta quindi di negare le considerevoli ingerenze delle ex potenze coloniali nei meccanismi politici, istituzionali, economici e culturali dei nuovi Stati indipendenti che finirono per limitarne effettivamente la sovranità, quanto piuttosto di riconoscere che questi meccanismi operarono sulla base di un'influenza indiretta, diversa dal sistema di dominio diretto coloniale.

\section{LA RINNOVATA POLITICA COLONIALE DELL'ITALIA NEL SECONDO DOPOGUERRA}

La fine del colonialismo italiano passò per quattro momenti diversi, ma fortemente interconnessi: la sconfitta dell'Italia in guerra con la perdita del dominio diretto sui possedimenti d'oltremare, ratificato poi dal Trattato di pace di Parigi nel 1947, e l'occupazione delle colonie da parte dell'Inghilterra e, limitatamente al Fezzan, da parte della Francia (1941-1947); la nascita e la rapida crescita dei movimenti indipendentisti e nazionalisti in Libia, Somalia ed Eritrea (1943-1950); la mancata approvazione del Compromesso Bevin-Sforza alle Nazioni Unite e le conseguenti decisioni prese dall'Assemblea Generale per una definitiva sistemazione delle ex colonie (1949-1950); il ritorno infine dell'Italia in Africa con un'amministrazione decennale diretta sulla Somalia (19501960). Queste quattro fasi descrivono una complessa transizione dal colonialismo all'indipendenza dei possedimenti italiani che può essere ricondotta alla più generale storia della decolonizzazione in Africa, con però un evidente elemento di specialità. Se di norma i processi di decolonizzazione si risolsero principalmente nel rapporto tra autorità coloniali e movimenti indipendentisti e nazionalisti, nel caso italiano il ruolo decisionale attribuito dal Trattato di pace di Parigi alle Quattro potenze vincitrici e poi alle Nazioni Unite, l'occupazione delle colonie da parte di Inghilterra e Francia e infine la rinnovata politica colonialista dell'Italia repubblicana relativizzarono il rapporto dialettico tra colonizzatori e colonizzati che normalmente costituì l'asse principale lungo il quale si svolsero i processi di decolonizzazione in Africa. La sconfitta dell'Italia in guerra non portò infatti all'immediata indipendenza delle

12 F. Cooper, Postcolonial Studies and the Study of History, in A. Loomba et al. (eds.), Postcolonial Studies and Beyond, Durham, Duke University Press, 2005, p. 403. 
colonie, ma a un'internazionalizzazione della questione della sistemazione delle ex colonie che fino al 1949 consistette nel tentativo di Inghilterra e Francia di sostituirsi al dominio dell'Italia, a sua volta impegnata nel riottenere i possedimenti d'Oltremare. È dunque difficile sostenere l'assenza di una dimensione propriamente coloniale in quello che fu l'ex impero italiano nel secondo lungo dopoguerra; piuttosto si può concludere per una sovrabbondanza e reciproca concorrenza dei diversi progetti coloniali di Italia, Inghilterra e Francia e delle loro azioni sul campo, in Africa, con l'eccezione rappresentata dall'Etiopia.

In questo quadro, va sottolineato come la sistemazione dell'Etiopia non fu mai parte delle discussioni internazionali proprio perché l'Impero etiopico non era mai stato ridotto allo status di una vera e propria colonia. Dopo aver difeso con successo la sua indipendenza contro l'aggressione coloniale italiana ad Adwa ai tempi del negusa nagast Menelik II nel 1896, l'Etiopia venne occupata nel 1935 durante la seconda guerra italo-etiopica. Dopo la caduta dell'Africa orientale italiana (Aoi) nel 1941, l'Impero etiopico venne subito restaurato nella propria sovranità internazionale sotto la corona dell'imperatore Haile Selassie per iniziativa della Gran Bretagna. L'Etiopia infatti era un paese sovrano aggredito e occupato dall'Italia fascista che, dopo la vittoria ad Adwa, si era visto riconoscere pienamente la sua sovranità internazionale entrando nel 1923 nella Società delle Nazioni. ${ }^{13}$ Il ritorno sul trono di Haile Selassie, dopo gli anni dell'esilio in Inghilterra, assunse d'altra parte i contorni di una restaurazione conservatrice proprio per coloro che erano rimasti a combattere in Etiopia e a lottare non solo per la liberazione dagli italiani, ma anche per una rivoluzione dello Stato e della società etiopica.

Nel secondo dopoguerra gli strumenti messi in campo da Inghilterra, Francia e Italia per perseguire i rispettivi piani coloniali furono differenti gli uni dagli altri, ma tutti conversero sull'obiettivo minimo della difesa del sistema coloniale, aggiornato alle forme del Trusteeship System delle Nazioni Unite. Gran Bretagna e Francia avevano intrapreso fin dagli anni della guerra una riforma dei loro sistemi imperiali in nome dello "sviluppo", inteso come benessere e crescita economica e sociale dei popoli colonizzati, superando così il concetto di

13 B. Zewde, A history of modern Ethiopia 1855-1991, Oxford, J. Currey, 2001. 
missione civilizzatrice che aveva costituito la legittimazione del colonialismo fin dall'Ottocento. La missione civilizzatrice aveva ridotto l'Africa a un continente eminentemente rurale e primitivo, fuori dal tempo e dalla storia, popolato da tribù, in contrapposizione con il mondo moderno e urbano dei colonizzatori. Non senza contraddizioni, i colonizzatori intendevano la loro missione nel senso di far "progredire" gli africani, ma attraverso l'etnografia produssero invece quell'apparato di conoscenze che finì per incapsulare le società africane in diversi gruppi etnici fissi e immutabili con l'intento di ordinarli in funzione dello stesso dominio coloniale. D'altra parte il colonialismo innescò anche una serie di processi di mobilità sociale che portarono via via quegli africani che più interagirono con il contesto economico e sociale europeo (non a caso definiti in termini peggiorativi "nativi detribalizzati") a contestare l'intrinseca disuguaglianza e gerarchia sociale dell'ordine coloniale e a rivendicare il benessere e il progresso promessi dai colonizzatori.

Proprio a seguito di una serie diffusa di scioperi e proteste sociali che sul finire degli anni Trenta percorsero le colonie inglesi in Africa, il governo di Londra decise di proclamare nel 1940 il Colonial Development and Welfare Act che «ruppe con la vecchia dottrina coloniale secondo la quale nessuna colonia avrebbe dovuto drenare risorse dal bilancio metropolitano» e si propose di finanziare con le risorse della madrepatria lo "sviluppo" e il benessere delle colonie. ${ }^{14}$ Così, «per almeno un decennio dopo il 1945, l'impero sembrò vitale per la Gran Bretagna, [... anzi] fino alla metà degli anni Cinquanta il sistema imperiale fu molto più importante per l'Inghilterra di quanto non lo fosse mai stato prima» e infatti, nonostante l'indipendenza della più importante colonia inglese, l'India, nel 1947, la maggior parte delle colonie africane divenne indipendente solo tra il 1957 e il $1964 .{ }^{15} \mathrm{Il}$ progetto inglese era quello di riformare l'impero per «conservare» il suo control$10^{16}$ e solo sul finire degli anni Cinquanta la progressione dei movimenti

14 F. Cooper, Development, Modernization, and the Social Sciences in the Era of Decolonization: The Examples of British and French Africa, in M. Bandeira Jeronimo, A. Costa Pinto (eds.), The Ends of European Colonial Empires. Cases and Comparisons, New York, Palgrave Macmillan, 2015, p. 22.

15 L.J. Butler, Britain and Empire. Adjusting to a Post-Imperial World, London, I.B. Tauris Publishers, p. 190.

16 R. Pearce, The Colonial Office and Planned Decolonization in Africa, African Affairs, 83, 330 (1984), p. 80. 
nazionalisti e le contraddizioni insite nelle riforme messe in atto «forzarono» l'Inghilterra a passare alla liquidazione del sistema imperiale. ${ }^{17} \mathrm{La}$ Gran Bretagna si impegnò allora in «una coerente e consapevole strategia di decolonizzazione» che andava nel senso di sostituire via via le native authorities con degli organi eletti, tra le cui pieghe esercitare un'influenza oltre l'indipendenza: in questa prospettiva i principali interlocutori di Londra divennero i nazionalisti, piuttosto che i vecchi capi e notabili.

Sulla scia di quanto avvenuto nel contesto inglese, il governo francese nel 1946 promosse la riforma che istituiva i Fonds d'investissement et développement économique et social, passando rapidamente dall'idea di assimilazione a quella di associazione dei popoli colonizzati. Si trattava pur sempre di agire di conserva e infatti «il concetto di sviluppo fu inteso come un problema tecnico che bisognava affrontare grazie a persone con una conoscenza specifica» la quale poteva arrivare solo dalle potenze coloniali e non certo dalle stesse società africane colonizzate: $:^{18}$ si passava così dal ruolo centrale svolto dagli etnografi all'inizio dell'impresa coloniale europea per lo studio delle società colonizzate per poi (ri)costruirle attraverso le categorie del sapere coloniale, a quella che è stata definita come una «seconda occupazione coloniale sotto forma di un'iniezione su larga scala di tecnici ed esperti» dello sviluppo intenti a rinnovare sotto nuove forme il meccanismo di dipendenza tra madrepatria e colonie. ${ }^{19}$ Il cambio dal primitivismo etnico al developmentalism, che ancora oggi anima i Development Studies, pose le premesse per fare della cooperazione allo "sviluppo" la più importante leva di interferenza politica nella nuova ottica dell'indipendenza. ${ }^{20} \mathrm{Fu}$ in nome di questa stessa «developmental ideology» letta al contrario che nella transizione finale all'indipendenza «le élite subentranti ai dominatori coloniali richiesero un governo forte formato da una casta governante convinta della sua superiore conoscenza». ${ }^{21}$

17 J. Flint, Planned Decolonization and Its Failure in British Africa, African Affairs, 82, 328 (1983), p. 411.

18 Cooper, Development, Modernization, and the Social Sciences, cit., p. 29.

19 J.D. Hargreaves, The End of Colonial Rule in West Africa, London, Macmillan, 1979, p. 41.

20 Ivi, p. 30.

21 C. Young, The Postcolonial State in Africa. Fifty Years of Independence, 19602010, Madison, University of Wisconsin Press, 2012, p. 337. 
In comparazione con gli sviluppi occorsi nei sistemi imperiali di Inghilterra e Francia, l'Italia nell'immediato dopoguerra si mosse lungo una direzione opposta, sostenendo l'idea di un ritorno alla missione civilizzatrice del primo colonialismo liberale. Per la nuova classe dirigente dell'Italia repubblicana se c'erano state delle colpe nel fallimento del progetto coloniale anteguerra, queste erano da attribuirsi al fascismo e non alla missione civilizzatrice della quale l'Italia si faceva appunto nuovamente carico. Il processo di auto-assoluzione dalle colpe del colonialismo rientrava nella più generale ripresa dei fili della storia patria che finì per caratterizzare gli italiani come «un popolo depositario di una grande civiltà, composto fondamentalmente da brava gente $i$ cui tratti essenziali non erano stati macchiati dal fascismo e neanche dalle sue guerre di aggressione» in Europa come in Africa. ${ }^{22}$ Lungo questo percorso interpretativo della storia recente prese corpo una delle narrazioni più consolidate intorno alla bontà degli italiani e del loro colonialismo, ossia la separazione e differenziazione del tutto capziosa e artificiale tra il "cattivo" colonialismo fascista e il "buon" colonialismo liberale.

In parlamento vi fu un'ampia convergenza tra le diverse forze politiche della Repubblica sulla legittimità delle rivendicazioni italiane verso le ex colonie al punto da assumere una portata simbolica di patria e italianità nella prospettiva tutt'altro che scontata della più generale ricostruzione della nazione italiana (oltre che dell'Italia). Pesarono le considerazioni di politica estera sulla "grandezza" dell'Italia e la mancata epurazione dell'amministrazione dello Stato: prevalse quella concezione dello "Stato amministrativo" che «privilegiava la valorizzazione del versante amministrativo rispetto a quello politico e [così] il ruolo delle burocrazie rimase a lungo forte». ${ }^{23}$ In questo senso, il caso italiano può essere accostato per più di un verso a quello del colonialismo tedesco e alla sua fine all'indomani della Grande guerra, sia per la richiesta, il $1^{\circ}$ marzo 1919, del parlamento della Germania di Weimar per «la restaurazione dei diritti coloniali tedeschi», sia per la conseguente nar-

22 S. Patriarca, Italianità. La costruzione del carattere nazionale, Roma-Bari, Laterza, 2010, pp. 217-218.

23 P. Pombeni, Le culture politiche nella transizione italiana: dalla guerra alla democrazia, in Formigoni e Saresella (a cura di), 1945. La transizione del dopoguerra, cit. p. 79. 
razione di un «colonialismo benevolo» che a buon diritto ambiva a ritornare in Africa. ${ }^{24}$

Con l'obiettivo di riportare l'Italia in Africa, ancora a guerra in corso, iniziò ad operare una lobby colonialista che fu in grado di mobilitare per lo scopo politici, funzionari coloniali, accademici ed esperti a vario titolo di Africa, coloni, concessionari e imprenditori che in Africa ancora risiedevano o vi avevano ancora interessi rilevanti. A tirare le fila di questa variegata lobby colonialista fu senza dubbio il Ministero dell'Africa italiana (Mai), ricostituito presso il palazzo della Consulta a Roma fin dal 15 luglio 1944, con un ruolo di raccordo istituzionale e di indirizzo dell'azione politica e diplomatica in favore del recupero dei possedimenti africani. L'opera del Mai si svolse in stretto coordinamento con il Ministero degli Affari esteri (Mae) e con una serie di altre istituzioni della galassia coloniale, come il Centro di studi coloniali di Firenze, la Società geografica africana d'Italia, l'Istituto di studi orientali, l'Istituto per l'Oriente, la Società africana d'Italia e l'Istituto coloniale (dal 1956 Istituto italo-africano), tutti finanziati attraverso il Mai. ${ }^{25}$

I piani italiani si scontrarono in Africa con la rapida crescita dei movimenti nazionalisti. L'abolizione da parte della British Military Administration (Bma) delle più restrittive tra le norme segregazioniste di epoca fascista portarono a un'ondata di liberazione nelle società colonizzate con la nascita delle prime associazioni, se non proprio partiti politici, che nei piani inglesi dovevano essere i nuovi intermediari della loro politica. Il Trusteeship System delle Nazioni Unite era chiamato ad assicurare lo strumento istituzionale più adatto a garantire la continuità del dominio coloniale, così come era stato alla fine della prima guerra mondiale del sistema dei mandati internazionali della Società delle Nazioni. ${ }^{26} \mathrm{Il}$ proget-

24 S. Conrad, German Colonialism. A Short History, Cambridge, Cambridge University Press, 2012, p. 186. In effetti «la campagna politica per ristabilire il dominio tedesco sulle colonie fece del "revisionismo" un obiettivo politico» che, una volta andato deluso, spinse il movimento coloniale ad appoggiare il Partito nazional-socialista. H. Pogge von Strandmann, The Purpose of German Colonialism, or the Long Shadow of Bismark's Colonial Policy, in V. Langbehn, M. Salama (eds.), German Colonialism. Race, The Holocaust, and Postwar Germany, New York, Columbia University Press, 2011, p. 206.

25 Non esiste in letteratura una stima d'insieme sui finanziamenti erogati attraverso il Mai. I dati d'archivio sono frammentari e aspettano ancora di essere ricomposti in una lettura complessiva.

26 A.M. Morone, L'Onu e l'Amministrazione fiduciaria italiana in Somalia. 
to italiano in comparazione con quello inglese risultava vecchio, perché proponeva una restaurazione dell'ordine coloniale addirittura del primo Novecento. Non a caso l'intesa che l'Italia cercò con i suoi ex sudditi si diresse soprattutto a ricercare l'appoggio dei gruppi più conservatori di quelle società, passate per il dominio italiano. In definitiva, per la nuova Italia repubblicana il colonialismo non finì in guerra: non solo l'Italia repubblicana esercitò una politica intesa ad affermare un dominio diretto, prettamente coloniale, in Africa, ma in tale contesto continuarono anche a funzionare specifiche istituzioni coloniali che dispiegarono le loro azioni tanto in Italia, quanto in Africa. Sulla base di queste due condizioni minime si può concludere per l'esistenza di un chiaro carattere coloniale, politico e istituzionale, della nuova Italia repubblicana nel secondo dopoguerra.

\section{VERSO UNA POLITICA POSTCOLONIALE}

Rispetto alla storia di lungo periodo del dominio italiano in Africa, la seconda guerra mondiale aveva fatto venir meno quella relazione speciale tra Inghilterra e Italia che alla fine dell'Ottocento aveva visto Londra appoggiare fattivamente l'espansione italiana al punto da mettere a disposizione di Roma la sua rete diplomatica e militare. L'acquisizione italiana della baia di Assab nel 1882 e poi l'occupazione del porto di Massawa nel 1885 lungo la costa del Mar Rosso avvenne grazie all'appoggio inglese che nel caso di Massawa prese la forma di un condominio italo-egiziano, ben sapendo che dal 1882 l'Egitto era diventato di fatto una colonia britannica; la cessione infine dei porti del Banaadir all'Italia, lungo la costa dell'Oceano indiano, da parte del sultano di Zanzibar venne negoziata nel 1892 grazie ai buoni uffici inglesi. L'Inghilterra aveva sempre visto con favore l'espansione di una piccola potenza come era l'Italia nel Corno d'Africa che non rappresentava una priorità strategica per Londra e dove tuttavia rimaneva importante evitare l'espansione di una grande potenza concorrente, come poteva essere il caso della Francia o della Germania. La seconda guerra mondiale aveva dunque trasformato due colonialismi complementari, come erano stati quello inglese e quello italiano, in con-

Dall'idea all'istituzione del trusteeship, Italia Contemporanea, 242, marzo (2006), pp. 45-64. 
correnti: forse proprio questo aspetto di lungo periodo sfuggiva alla diplomazia italiana quando si interrogava sull'ostinazione inglese, specie del Colonial Office, a mantenere una posizione avversa all'Italia anche dopo la fine della guerra e la firma del Trattato di pace. Sta di fatto che tanto a Londra quanto a Roma, la sistemazione delle ex colonie italiane venne interpretata soprattutto come una questione principalmente interna al rapporto tra Italia e Inghilterra.

Le dirigenze dei due paesi pensarono di aver raggiunto una soluzione complessiva per le ex colonie attraverso un'intesa segreta bilaterale, il Compromesso Bevin-Sforza, siglato il 6 maggio 1949, non a caso a seguito delle elezioni dell'aprile 1948 e dell'adesione dell'Italia alla North Atlantic Treaty Organization (Nato). Entrambi questi passaggi avevano infatti la valenza di una scelta di campo dell'Italia alla quale Gran Bretagna e Stati Uniti avevano sempre subordinato ogni possibile soddisfazione in campo coloniale. ${ }^{27}$ Il Compromesso delineava una spartizione prettamente coloniale dei possedimenti italiani che infatti non teneva in considerazione le istanze delle popolazioni delle ex colonie: la Libia veniva divisa e sottoposta all'amministrazione fiduciaria dell'Inghilterra in Cirenaica, dell'Italia in Tripolitania e della Francia nel Fezzan; l'Eritrea veniva divisa tra la regione dell'altopiano annessa all'Etiopia e quella del bassopiano incorporata invece nel Sudan inglese, mentre uno status particolare era previsto per le città di Asmara e Massawa al fine di tutelare gli interessi e le specificità delle comunità italiane ancora colà residenti; la Somalia tornava invece all'Italia in amministrazione fiduciaria. In definitiva, il Compromesso rappresentava una soluzione per i diversi e contrapposti progetti coloniali europei sul destino degli ex possedimenti italiani, non certo una soluzione per le istanze indipendentiste dei movimenti nazionali.

Il vero punto di svolta nella complessa vicenda della sistemazione delle ex colonie italiane arrivò il 18 maggio 1949, quando l'Assemblea generale dell'Onu non approvò il Compromesso Bevin-Sforza. A far mancare il quorum all'Assemblea Generale dell'Onu fu nello specifico il voto del rappresentante di Haiti, proprio uno dei "paesi amici" del

27 G.P. Calchi Novati, Studi e politica ai convegni coloniali del primo e del secondo dopoguerra, in Fonti e problemi della politica coloniale italiana, Atti del Convegno Taormina-Messina, 23-29 ottobre 1989, vol. 1, Roma, Istituto Poligrafico dello Stato, 1996, pp. 192-193. 
gruppo latino-americano che avrebbero dovuto sostenere le posizioni italiane, ma più in generale le ragioni della mancata approvazione andavano ricondotte a un sistema internazionale in forte e rapido cambiamento dove contava sempre di più il ruolo giocato in Assemblea Generale dai paesi di nuova indipendenza e le logiche di influenza indiretta delle due superpotenze. Con il fallimento dell'ultimo tentativo di mantenere sotto il regime coloniale le ex colonie, Italia e Gran Bretagna si dissero allora pronte a sostenerne l'indipendenza. Il ministro Sforza si recò al Comitato politico delle Nazioni Unite il $1^{\circ}$ ottobre 1949 sostenendo per la prima volta dalla fine della guerra che l'Italia si impegnava per l'indipendenza immediata di Libia ed Eritrea e, dopo un'adeguata preparazione, per quella della Somalia. Rispetto alle rivendicazioni coloniali del primo dopoguerra, si trattava di una vera capriola diplomatica che perseguiva pur sempre «un fondo tattico» nella convinzione che l'influenza dovesse essere esercitata a questo punto attraverso (e non contro) l'indipendenza delle colonie. ${ }^{28}$ Il mandato trentennale che era stato chiesto sulla Somalia in un'ottica rivolta ancora al passato, venne riformulato con l'obiettivo di contribuire allo sviluppo del paese in funzione di raggiungere l'indipendenza nel giro di un decennio. A pochi mesi dalla fine del mandato internazionale e dall'indipendenza della Somalia erano indicativamente gli Stati Uniti a invitare gli italiani ad assumersi in pieno le loro responsabilità poiché, come riferiva l'ambasciatore d'Italia a Washington, «maggiori saranno i nostri controlli sull'economia somala, più mezzi avremo per resistere alle pressioni di Mogadiscio» verso una politica autonoma e potenzialmente aperta al blocco sovietico, perciò in Somalia «occorreva conservare sia di fronte al nuovo Stato, sia di fronte agli alleati, la nostra piena influenza». ${ }^{29}$ L'opera dell'Italia in Somalia doveva allora testimoniare il nuovo impegno dell'Italia per le indipendenze africane, non solo della Somalia.

L'opportunismo della svolta italiana, che prefigurava un passaggio da una politica intesa a recuperare un dominio diretto a una indirizzata piuttosto a ricercare un'influenza indiretta, lasciava ai movimenti nazio-

28 G.P. Calchi Novati, La sistemazione delle colonie italiane dell'Africa Orientale e i condizionamenti della guerra fredda, in A. Del Boca (a cura di ), Le guerre coloniali del fascismo, Roma-Bari, Laterza, 1991, p. 533.

29 Archivio Storico Diplomatico del Ministero degli Affari Esteri (d'ora in poi ASDMAE), Affari Politici (d'ora in poi AP), b. 247, f. 840, lettera da Brosio al Mae, 19 febbraio 1960. Manlio Brosio era ambasciatore d'Italia a Washington. 
nalisti il dubbio, più che giustificato, che si trattasse di una svolta tutt'altro che genuina. L'Italia cercò di proporsi come un partner privilegiato dei nuovi Stati indipendenti del Corno d'Africa e dei paesi arabi nel quadro dell'Alleanza atlantica, facendo perno sui crediti maturati attraverso la sua nuova politica a favore dell'indipendenza dell'Africa, che tuttavia rischiavano di essere pochi e non del tutto spendibili proprio a causa degli anni persi nella rinnovata politica coloniale durante il secondo dopoguerra e di una mancata condanna in modo chiaro del passato coloniale. Il banco di prova per questa nuova politica fu l'esperimento dell'Amministrazione Fiduciaria Italiana della Somalia (Afis).

Se il colonialismo europeo tra Otto e Novecento è stato definito prima di tutto in termini di occupazione ed esercizio diretto del dominio, anche nei casi dei protettorati che salvano più le forme che la sostanza del potere precoloniale, allora solo il riconoscimento dell'indipendenza delle statualità africane apriva alla decolonizzazione e in prospettiva alla fine del colonialismo. Dunque il 1949 fu il vero tornante storico, specie se si fa lo sforzo di non appiattire tutta l'analisi sul versante italiano della storia e di considerare la storia del colonialismo come parte della storia dell'Africa. Si può allora concordare con Nicola Labanca che il colonialismo italiano ebbe «diverse fini, diversi processi di decolonizzazione» a seconda di quale ex colonia si consideri ${ }^{30}$ come peraltro si può dire qualcosa di molto simile per la gran parte delle colonie in Africa. Tuttavia occorre sottolineare come questi diversi processi di decolonizzazione discendano tutti dalla svolta del 1949. Inoltre, dando per acquisito questo quadro composito e articolato, se si è disposti a ritenere il 1882 l'anno «di nascita del colonialismo» italiano, quando l'allora Regno d'Italia acquistò la concessione di Assab dalla compagnia di navigazione genovese Rubattino e prese possesso solo di un piccolissimo avamposto all'interno di quello che poi divenne nel corso dei decenni successivi lo spazio coloniale italiano, ${ }^{31}$ allora si deve essere anche disposti a riconoscere che l'ultima amministrazione diretta dell'Italia in Africa venne liquidata con l'indipendenza della Somalia solo nel 1960. In estrema sintesi, se il colonialismo italiano iniziò nel 1882 lungo le rive del Mar Rosso, finì nel 1960 in Somalia, lungo le coste dell'Oceano indiano. Nella prospettiva della "ultima colonia"

30 Labanca, Oltremare, cit., p. 434.

31 Calchi Novati (a cura di), L'Africa d'Italia, cit., p. 386. 
somala, la parabola del dominio italiano in Africa traccia dunque un arco cronologico in piena consonanza con la fine della maggior parte degli altri colonialismi europei, in quel 1960 passato alla storia come "l'anno dell'Africa," quando ben 17 paesi africani divennero indipendenti e l'Assemblea generale dell'Onu approvò il 14 dicembre la famosa risoluzione 1514 in favore dell'autodeterminazione dei paesi e dei popoli colonizzati. Dal 1949 l'Italia repubblicana fu dunque impegnata in tre diversi processi di decolonizzazione, due molto rapidi e uno più lungo gestito direttamente dall'Italia: proprio il caso somalo rivela bene come la transizione verso l'indipendenza non fu affatto un processo univoco, ma passò per il progressivo affermarsi del nuovo sulle molte vischiosità e resistenze della vecchia politica coloniale e delle sue istituzioni. In conclusione, la fine del colonialismo in Libia e nel Corno d'Africa si caratterizzò per una concorrenza tra diversi progetti coloniali e non certo per una rapida "ritirata" del dominio europeo.

\section{LE EREDITÀ COLONIALI}

Nella prospettiva della storia dell'Africa, la transizione all'indipendenza della Libia e il riconoscimento dell'autonomia eritrea nell'ambito della federazione con l'Etiopia avvennero in anticipo rispetto all'anno dell'Africa, il 1960. La differente cronologia libica ed eritrea si combinò anche con un differente esito della liquidazione del sistema coloniale nel senso che tanto la monarchia libica quanto la federazione etiopico-eritrea nacquero con al vertice dello Stato una classe composta essenzialmente dai dirigenti di epoca coloniale o precoloniale. Quelli che con un termine molto generale furono definiti "capi" all'interno dell'amministrazione coloniale ebbero in realtà una fisionomia sociale $\mathrm{e}$ politica molto variegata: a volte si trattò di vecchi dirigenti inglobati nel nuovo sistema di gestione coloniale; in altri casi furono figure antagoniste agli assetti di potere consolidati a essere appoggiate perché meglio disposte a intermediare con il potere coloniale; altri capi ancora furono letteralmente inventati e intronizzati nell'ambito di società che non conoscevano figure di quel tipo. Le autorità africane intermediarono con il potere coloniale, piuttosto che collaborarvi, nel senso che la relazione esistente fu molto spesso di scambio e contrattazione reciproca, piuttosto che di semplice servizio al potere coloniale. Tra le pieghe della decolonizzazione, le native authorities dell'impero inglese, i chefs dei 
distretti amministrativi dell'impero francese o i capi-qabila dell'impero italiano cercarono di difendere le loro posizioni di potere in un quadro politico e sociale in forte mutamento.

La diffusione del nazionalismo in Africa pose i vecchi capi coloniali in una posizione oggettivamente difficile e controversa. Le nuove élites nazionaliste lottarono per l'indipendenza, scommettendo su una mobilitazione a carattere nazionale, contro le identità localistiche che costituivano il quadro di riferimento sociale dei loro diretti competitori politici: «Chi privilegiava una regione o una comunità o una monarchia nel frattempo integrata in una colonia o in un protettorato passava automaticamente per separatista o secessionista ed era messo al bando [... attraverso] una reinvenzione di se stessi nel tentativo di rimediare agli inganni dell'invenzione altrui». ${ }^{32}$ Lo Stato coloniale, che doveva fare gli interessi degli europei e di pochi intermediari, andava riformato in funzione della nazione che rimandava a un'idea di interesse comune e a un concetto di "sviluppo" inteso prima di tutto come welfare. I nazionalisti avevano fatto propria l'agenda sviluppista dopo gli insuccessi dei progetti postbellici di riforma dell'impero britannico e francese.

Nel caso del sistema inglese, il riconoscimento di un certo ambito di autonomia di gestione non solo amministrativa, ma anche politica alle native authorities fu determinante nel preservare loro una residuale legittimità politica e un certo prestigio sociale, anche se proprio nel rapporto con i nazionalisti andò crescendo a partire dal secondo dopoguerra l'opposizione a un ordine coloniale con il quale quelle stesse autorità erano profondamente implicate. Nel sistema francese, belga e anche in quello italiano, pur con alcune differenze, i capi erano figure prevalentemente amministrative e, applicando gli ordini dei funzionari europei, finirono per essere ampiamente screditati agli occhi delle loro società di appartenenza. ${ }^{33}$ In ogni caso la figura del capo, del dirigente africano, inserito nell'amministrazione coloniale era andata trasformandosi in modo rilevante e dunque risultava intrinsecamente diversa da quella dei capi precoloniali, al netto di tutte le differenze tra i diversi sistemi di amministrazione in colonia. Per questo motivo il riferimento a una contrapposizio-

32 G.P. Calchi Novati, Dalla parte dei leoni. Africa nuova, Africa vecchia, Milano, Il Saggiatore, 1995, pp. 76-77.

33 Sul caso dell'amministrazione coloniale italiana, A.M. Morone, Un quadro istituzionale ibrido, in Calchi Novati (a cura di), L'Africa d'Italia, cit., pp. 213-235. 
ne tra moderno e tradizionale con riferimento rispettivamente ai partiti nazionali e ai capi inseriti nell'amministrazione coloniale rischia di essere semplicistico: vecchi capi e giovani nazionalisti appartenevano alla stessa società coloniale, pur provenendo da ambiti e storie diverse, e allora il "tradizionale" non poteva essere il precoloniale.

I nazionalisti lottavano per «il diritto di autodeterminazione»e per creare un'unità politica «indipendente o almeno fortemente autonoma», mentre i capi aspiravano piuttosto a una progressione del sistema coloniale che garantisse loro «l'auto-conservazione e un miglioramento sociale ed economico» nella prospettiva di quei gruppi etnici nei quali il sapere coloniale li aveva confinati e in parte (ri)costruiti: in questi termini si può intendere lo scarto tra un'agenda nazionale e un'agenda etnica, ${ }^{34}$ anche se l'opzione conservatrice non implicava automaticamente una presa di posizione contraria all'indipendenza. Non mancarono infatti casi di capi che con l'incedere della decolonizzazione lottarono attivamente per il suo conseguimento. In questo caso la differenza con i nazionalisti restava nei contenuti che si voleva dare a quell'indipendenza: i nazionalisti guardavano a un'indipendenza progressista se non proprio rivoluzionaria, che mettesse in discussione gli assetti politici e sociali del passato regime coloniale, mentre i capi aspiravano a un'indipendenza conservatrice che garantisse in ogni caso le loro posizioni di potere. Una tale contrapposizione tra vecchio e nuovo si caratterizzò anche nei termini di un contrasto generazionale tra il conservatorismo sociale dei vecchi intermediari e una nuova visione progressista dei giovani nazionalisti.

Guardando al continente africano, furono spesso i nazionalisti ad avere la meglio sui capi e notabili, esponenti di quell'ordine conservatore che proprio il progetto nazionale aspirava a rivoluzionare. I dirigenti dei nuovi Stati africani provenivano da quelle élites di évolués che erano andate formandosi, istruendosi e rafforzandosi nell'interazione con i dominatori coloniali, assimilandone progressivamente tratti della cultura, della società e del sapere moderno. Si trattava di persone nuove, che avevano in molti casi intermediato anch'essi con il potere coloniale ma su posizioni diverse rispetto a quelle dei vecchi dirigenti pre-coloniali, cooptati nell'amministrazione delle potenze europee o a

34 C. Young, The Postcolonial State in Africa. Fifty Years of Independence, 19602010, Madison, University of Wisconsin Press, 2012, p. 330-1. 
quelli direttamente intronizzati dalle stesse potenze coloniali. La storia dei vecchi capi e notabili coloniali si può allora considerare come, in gran parte, la storia degli sconfitti. Furono in effetti poche le eccezioni, tra le quali però spiccano i casi di Idris al-Sanusi e di Haile Selassie. ${ }^{35}$ In Libia e nella federazione tra Etiopia ed Eritrea non furono i nazionalisti a guidare gli Stati indipendenti, bensì i vecchi dirigenti che avevano lottato contro il colonialismo o vi avevano intermediato oppure entrambe le cose e che riuscirono ad aggiornarsi con successo al nuovo contesto post-coloniale. La Somalia rientrò invece nella più generale e "normale" tipologia delle decolonizzazioni che videro i nazionalisti prendere il potere dello Stato indipendente proprio perché in Somalia l'Italia, ritornando in colonia, predispose una transizione all'indipendenza dialogando con i nazionalisti somali. I dieci anni dell'Afis rappresentarono l'unica vera decolonizzazione gestita in autonomo dall'Italia, al di là dei paletti piuttosto laschi imposti dal Consiglio di tutela delle Nazioni Unite: appare così evidente come gli anni Quaranta nel caso somalo furono una parentesi di un dominio coloniale altrui, quello britannico, all'interno di una più lunga storia di dominio italiano fino all'indipendenza della Somalia il $1^{\circ}$ luglio $1960 .{ }^{36}$ La decolonizzazione della Libia e la federazione dell'Eritrea all'Etiopia in un momento storico nel quale le grandi e le piccole potenze coloniali non avevano ancora rinunciato all'idea dell'impero favorì una convergenza impropria tra le vecchie élites conservatrici e i progetti di influenza indiretta delle ex potenze coloniali. Ne conseguì la sconfitta dei nazionalisti che ebbero poi la loro rivincita con la Rivoluzione di Mu'ammar al-Qadhdhafi nel 1969 in Libia e quella del Derg nel 1974 in Etiopia.

35 Ricordo inoltre in Marocco, Muhamad ibn Yusuf (re Muhamad V dal 1957); nel Burundi, mwami Mwambutsa IV; nel Lesotho, Moshoeshoe II; nello Swaziland, Sobhuza II.

36 A.M. Morone, L'ultima colonia. Come l'Italia è tornata in Africa, 1950-1960, Roma-Bari, Laterza, 2011. 